\title{
Risk Factors of Pleural Effusion Following Pediatric Liver Transplantation and the Perioperative Outcomes
}

This article was published in the following Dove Press journal:

Transplant Research and Risk Management

\author{
Chollasak Thirapattaraphan ${ }^{1,2}$ \\ Prapatsorn Srina $\mathbb{D}^{\prime}$ \\ Ampaipan Boonthai $\mathbb{D}^{1,2}$ \\ Nuttapon Arpornsujaritkun 2,3 \\ Bundit Sakulchairungrueng (D) ${ }^{2,3}$ \\ Worapot Apinyachon ${ }^{2,4}$ \\ Suporn Treepongkaruna $\mathbb{D}^{2,5}$
}

'Division of Pediatric Surgery, Department of Surgery, Faculty of Medicine Ramathibodi Hospital, Mahidol University, Bangkok, Thailand;

${ }^{2}$ Ramathibodi Excellent Center for Organ Transplantation, Faculty of Medicine Ramathibodi Hospital, Mahidol University, Bangkok, Thailand; ${ }^{3}$ Division of Vascular Surgery and Transplantation, Department of Surgery, Faculty of Medicine Ramathibodi Hospital, Mahidol University, Bangkok, Thailand;

${ }^{4}$ Department of Anesthesiology, Faculty of Medicine Ramathibodi Hospital, Mahidol University, Bangkok, Thailand;

${ }^{5}$ Division of Gastroenterology, Department of Pediatrics, Faculty of Medicine Ramathibodi Hospital, Mahidol University, Bangkok, Thailand
Background: Pediatric liver transplantation (LT) has been accepted as a definitive treatment for end-stage liver disease. Pleural effusion is a common pulmonary complication following LT in children. The objectives of the study were to identify prevalence of post-LT pleural effusion, risk factors, and the impact on patients' outcomes.

Methods: A retrospective study was conducted in 107 pediatric patients who underwent LT at our center between March 2001 and June 2018. They were categorized into pleural effusion and non-pleural effusion groups. Preoperative and perioperative data, intraoperative findings, liver graft characteristics, and perioperative outcomes were compared between the two groups.

Results: Post-LT pleural effusion occurred in 64 (59.8\%) patients. There were more patients with PELD score $\geq 18$ in the pleural effusion group (68.8 vs $48.8 \%, P=0.039$ ). Other preoperative and perioperative data were not significantly different. The pleural effusion group had a higher rate of reoperation than non-pleural effusion group (55.6 vs $30.9 \%$, $P=0.013$ ). Median oxygen dependence time, length of ICU and hospital stay were significantly longer in the pleural effusion group (18.5 vs 7.0, 10 vs 7 and 48 vs 34 days, respectively, $P<0.05)$. However, mortality was not significantly different. Among the patients with pleural effusion, median time to extubation, oxygen dependence time, length of ICU and hospital stay were significantly longer in those who required therapeutic interventions than those without interventions (12 vs 3,31 vs 10,17 vs 8 , and 60 vs 43 days, respectively, $P<0.05)$.

Conclusion: Pleural effusion following pediatric LT is common and its potential risk factor is PELD score at LT $\geq 18$. Post-LT pleural effusion is associated with prolonged oxygen dependence time, ICU stay and hospital stay, particularly those who required therapeutic interventions. Keywords: pleural effusion, pediatric liver transplantation, liver transplantation, post-liver transplant pleural effusion, pulmonary complications

\section{Introduction}

Liver transplantation (LT) has been accepted as a definite treatment for acute liver failure and end-stage liver disease. Pulmonary complications following LT, including pleural effusion, pulmonary edema, atelectasis and pneumonia have been commonly reported both in adults ${ }^{1-4}$ and children. ${ }^{5-8}$ These complications increase morbidity, prolong mechanical ventilation, intensive care unit (ICU) stay and hospitalization, and significantly reduce short-term survival. ${ }^{1,2,9,10}$ Pleural effusion has been reported as the most common pulmonary complication with the prevalence
Correspondence: Suporn Treepongkaruna Division of Gastroenterology, Department of Pediatrics, Faculty of Medicine Ramathibodi Hospital, Mahidol University, 270 Rama VI Road, Ratchathewi, Bangkok 10400, Thailand

$\mathrm{Tel} / \mathrm{Fax}+66220 \mathrm{II} 446$

Email suporntr@gmail.com
Transplant Research and Risk Management 2020:I2 29-35 
of $32-47 \%$ in adult LT recipients. ${ }^{2-4,7}$ Pleural effusion develops mainly in the right side during the first week after LT but most do not require therapeutic interventions such as thoracentesis and chest tube placement. ${ }^{2,6}$ Although it is generally a non-serious self-limiting condition, large amount and prolonged course of pleural effusion can result in atelectasis and compromised respiratory status particularly in young children. ${ }^{2,11}$ The commonly identified risk factors for post-operative pulmonary complications include high Pediatric End-Stage Liver Disease (PELD) or Model for End-Stage Liver Disease (MELD) score, acute rejection, positive fluid balance, massive transfusion of blood products, and hypoalbuminemia. ${ }^{2,5-8}$ However, risk factors associated with post-LT pleural effusion have been rarely reported. ${ }^{5,12}$ We therefore conducted a study with the aim to determine the prevalence of postLT pleural effusion, risk factors, treatment and its impact on early post-LT outcome in the pediatric LT center.

\section{Patients and Methods}

We retrospectively reviewed patients aged 18 years or less who underwent LT at the Faculty of Medicine Ramathibodi Hospital, Mahidol University, Thailand, during the period between March 2001-June 2018. Of the total 140 patients, we excluded 33 children due to missed and/or incomplete medical records, therefore 107 patients were included. The patients were categorized into pleural effusion and non-pleural effusion groups. Post-LT pleural effusion was defined as pleural effusion diagnosed by either plain chest film, ultrasound, or CT/MRI within 3 months post-LT. At our center, daily plain chest film was routinely performed until the patients were successfully extubated without pulmonary symptoms. Lung ultrasound and CT/MRI were performed only when clinically indicated.

Patient characteristics, preoperative data, graft characteristics and outcomes were reviewed in both groups. The patients' demographic data included complications of endstage liver disease, preoperative pulmonary diseases, serum albumin level and PELD/MELD score for patients aged below 12 years old and $\geq 12$ years old, respectively. Perioperative data included graft type, graft-to-recipient weight ratio, presence of ascites identified by the surgeon, operative time, intraoperative blood loss and fluid balance. Postoperative data included fluid balance in the first 3 days post-LT and postoperative complications. Management of pleural effusion and interventions and, if applicable, the profiles of pleural fluid and duration of drainage were collected.

The patients were routinely admitted in the pediatric ICU after operation until they were successfully extubated with stabilized cardiopulmonary status before being transferred to pediatric surgical ward. Oxygen dependence time was defined as duration from LT to the day of discontinuing oxygen therapy. Other pulmonary complications such as atelectasis, hemo/pneumothorax, chylothorax and pneumonia were also recorded.

The study was approved by the Ethics Committee for Human Research, Faculty of Medicine Ramathibodi Hospital, Mahidol University. Written informed consent for a retrospective study was not required after the approval from the Ethics Committee for Human Research in our institution because there were no interventions applied to the patients. The study complied with the Declaration of Helsinki and the individual data of all patients were kept confidential. Organs were donated voluntarily with written informed consent, and donations were conducted in accordance with the Declaration of Istanbul.

\section{Statistical Analysis}

Data were presented as mean, median (IQR) or percentage. Patient data between two groups were compared using the Student's $t$-test or Mann-Whitney test for continuous variables, and Chi squared or Fisher's exact test for categorical variables. $P<0.05$ was considered statistically significant. All analyses were performed with STATA version 14 (StataCorp LLC, USA).

\section{Results}

Of the 107 patients, 41 were males $(38.3 \%)$ and the median (range) age at LT was 17.5 (3-204) months. The most common indication for LT was cirrhosis secondary to biliary atresia (78.5\%). One hundred and five patients (98.1\%) received partial liver grafts (mostly left lateral segment of left lobe) from living donors. Two patients received whole liver grafts from cadaveric donors.

Post-LT pleural effusion was found in 64 patients (59.8\%). Patient characteristics and pre-LT data of the pleural effusion and non-pleural effusion groups are shown in Table 1. None had underlying chronic pulmonary diseases before LT. Two patients had comorbidity of inactive congenital heart disease. There were more patients with PELD score $\geq 18$ in the pleural effusion group than the other ( 68.8 vs $48.8 \%, P=0.039$ ), while the other preoperative comorbidities including a presence of 
Table I Comparison of Demographic Data and Preoperative Clinical Parameters Between Pleural Effusion and Non-Pleural Effusion Groups

\begin{tabular}{|c|c|c|c|}
\hline Data & Pleural Effusion $(n=64)$ & Non-Pleural Effusion $(n=43)$ & $P$-value \\
\hline Male (n, \%) & $35(54.7)$ & $31(72.1)$ & 0.072 \\
\hline Age at LT (months); median (IQR) & $16.0(13.0,30.0)$ & $21.5(13.0,37.0)$ & 0.900 \\
\hline BW at LT $(\mathrm{kg})$, median (IQR) & $8.6(7.3,10.1)$ & $9.9(7.5,12.0)$ & 0.888 \\
\hline PELD score at LT, median (IQR) & $20.1(16.5,24.1)$ & $17.9(14.4,22.0)$ & 0.068 \\
\hline PELD score at LT $\geq 18, \mathrm{n}(\%)$ & $44(68.8)$ & $21(48.8)$ & $0.039 *$ \\
\hline \multicolumn{4}{|l|}{ Underlying liver diseases, n (\%) } \\
\hline Biliary atresia & $50(78.1)$ & $34(79.1)$ & 0.917 \\
\hline Neonatal hepatitis & $3(4.7)$ & $2(4.7)$ & 0.972 \\
\hline Budd Chiari syndrome & I (I.6) & $0(0.0)$ & - \\
\hline Alagille syndrome & $2(3.1)$ & I (2.3) & 0.792 \\
\hline Metabolic liver disease & $\mathrm{I}(\mathrm{I} .6)$ & $\mathrm{I}(2.3)$ & 0.789 \\
\hline Acute liver failure & $3(4.7)$ & 0 & - \\
\hline Cirrhosis unknown cause & $3(4.7)$ & $3(6.9)$ & 0.637 \\
\hline Others & I (I.6) & $2(1.9)$ & 0.343 \\
\hline \multicolumn{4}{|l|}{ Pre-LT comorbidities, n (\%) } \\
\hline Portal hypertension & $23(35.94)$ & II (25.6) & 0.261 \\
\hline SBP & $3(4.7)$ & $2(4.7)$ & 0.972 \\
\hline Pneumonia & $3(4.7)$ & $2(4.7)$ & 0.972 \\
\hline Hepatic encephalopathy & $2(3.1)$ & $\mathrm{I}(2.3)$ & 0.792 \\
\hline Upper Gl bleeding & $2(3.1)$ & $3(7.0)$ & 0.972 \\
\hline Pre-LT ascites, n (\%) & $48(75.0)$ & $27(62.8)$ & 0.176 \\
\hline Pre-LT serum albumin (gram/L), median (IQR) & $26.2(22.8,31.0)$ & $25.8(23.0,31.7)$ & 0.573 \\
\hline
\end{tabular}

Note: $* P<0.05$ was statistically significant.

Abbreviations: LT, liver transplant; SBP, spontaneous bacterial peritonitis.

preoperative ascites were not significantly different between the two groups. The ROC showed PELD score of 18 provides a fair discriminating capacity $(0.60)$ for post-LT pleural effusion with the sensitivity and specificity of 68.7 and 51.2\%, respectively (Figure 1).

The median volume of blood component transfusion in the pleural effusion group was insignificantly higher than the non-pleural effusion (170.3 vs $114.1 \mathrm{~mL} / \mathrm{kg}$, $P=0.054)$. Other operative parameters, intraoperative and postoperative fluid balance in the first 3 days were not significantly different between the two groups (Tables 2 and 3). Postoperative complications were not different between the two groups, however, bile duct complications and reoperation rate were significantly higher in the pleural effusion group (Table 4). Length of ICU stay, hospital stay and oxygen dependence time were significantly longer in the pleural effusion group than in non-pleural effusion group (Table 5).

Pleural effusion was mainly noted in the right side (78.1\%). Mean (SD) onset of pleural effusion was 7.0
(6.4) days post LT. Therapeutic interventions were performed in 24 cases $(31.3 \%)$ including intercostal tube drainage (ICD) $(n=20)$ and thoracocentesis $(n=4)$. Median duration (IQR) of ICD was $11.5(7,19)$ days. Among the patients with pleural effusion, median time to extubation, oxygen dependence time, length of ICU and hospital stay were significantly longer in those who required therapeutic interventions than in those without interventions (Table 6).

Of the 24 patients who had pleural fluid examinations, the profiles were compatible with exudate $(\mathrm{n}=19,79.2 \%)$, transudate $(n=3,12.5 \%)$, chylothorax $(n=1,4.2 \%)$ and 1 missing data. Other pulmonary complications were not significantly different: atelectasis $(28.1 \%$ in the pleural effusion group vs $37.2 \%$ in the non-pleural effusion group, $P=0.32$ ) or pneumonia ( $1.5 \%$ vs $4.6 \%, P=0.37)$.

Six patients, three in each group, died. Causes of death included primary graft non-function $(n=2)$, bacterial pneumonia $(n=1)$, peritonitis $(n=1)$, and septicemia $(n=2)$. None were related to pleural effusion. 


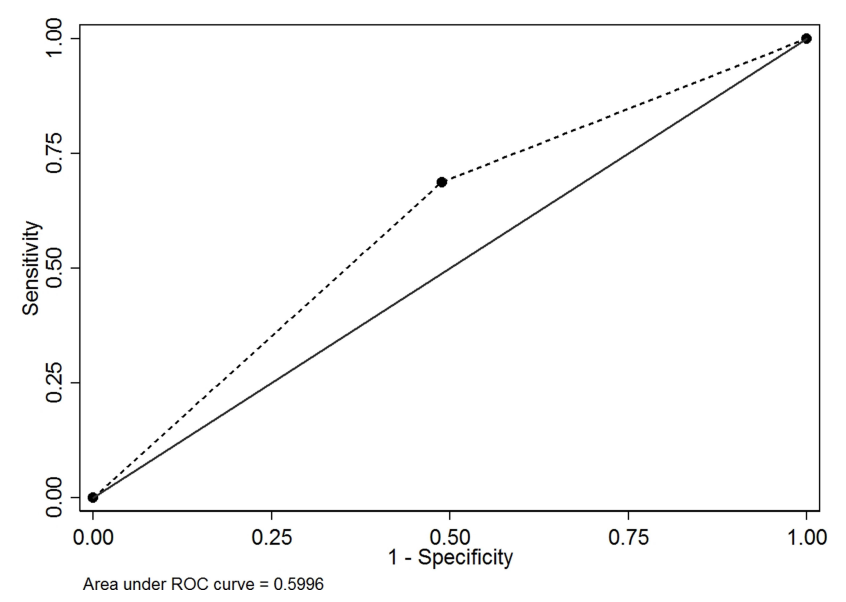

Figure I The association between pre-transplant Pediatric End-Stage Liver Disease (PELD) score and post-transplant pleural effusion. PELD score of 18 provides a fair prediction capacity of 0.60 with the sensitivity and specificity of 68.7 and 51.2 , respectively.

\section{Discussion}

Our study showed that the prevalence of pleural effusion following pediatric LT was relatively high (59\%), and most occurred in the right lung. Although effusion is generally self-limiting, approximately one-third of the patients in this study required therapeutic interventions including ICD and thoracocentesis. Patients with postLT pleural effusion had prolonged oxygen dependence time, length of ICU stay and hospital stay when compared to those without pleural effusion. Moreover, our study demonstrated that patients with pleural effusion who required therapeutic interventions had longer time to extubation, oxygen dependence time, length of ICU stay and hospital stay when compared with those who did not require any interventions. Patients in our study had relatively long ICU and hospital stay, which was likely due to the high rate of post-LT complications in both groups (93.7 and $83.7 \%$, respectively). Moreover, the pleural effusion group had a higher rate of reoperation, mainly due to surgical complications which could also have contributed to the longer ICU and hospital stay.
Prevalence of post-LT pleural effusion in this study was slightly higher than in previous reports. RuchonnetMetrailler et $\mathrm{al}^{5}$ reported post-LT pleural effusion $<7$ and $\geq 7$ days occurred in $37 \%$ and $28 \%$ of 79 children, respectively. Effusion $\geq 7$ days was associated with low serum albumin level and respiratory distress or hypoxia at admission. ${ }^{5}$ Alam et $\mathrm{al}^{6}$ showed that pleural effusion developed in $49 \%$ of 45 LT recipients in which $18 \%$ required thoracocentesis or tube thoracostomy. Sato et $\mathrm{al}^{8}$ reported a prevalence of $52 \%$ in 41 pediatric LT recipients. Adetiloye and John ${ }^{11}$ reported 23 of the 184 pediatric LT $(12.5 \%)$ developed a large amount of pleural effusion requiring therapeutic interventions. Mean duration from treatment to resolution was 33 days. ${ }^{11}$ Wiering et al ${ }^{12}$ have reported risk factors of post-LT pleural effusion requiring ICD in adult recipients include donor and recipient body mass index (BMI), hospitalized status, serum urea and number of intraoperative red blood cell transfusions.

Our study showed that high preoperative PELD/MELD score $\geq 18$ was the only factor associated with post-LT pleural effusion. However, the ROC provided a fair discriminating capacity of 0.60 (Figure 1). Pediatric endstage liver disease (PELD) score is a good measurement for defining the severity of chronic liver disease that would predict mortality of children awaiting LT and post-LT survival. $^{13,14}$ In fact, PELD score of $\geq 25$ was shown to be an independent risk factor for developing pulmonary complications following pediatric LT. ${ }^{6}$ Similarly, high MELD score increased the risk of pulmonary complications after LT in adult studies. ${ }^{2}$ Our patients with high PELD score had severe portal hypertension with multiple collateral vessels and, according to our experience, it was often complicated by massive intraoperative bleeding resulting in increased intraoperative and postoperative fluid resuscitation. We noted that our patients with pleural effusion received more blood component transfusions than the non-pleural effusion group, although it did not reach statistical significance. This could contribute to an

Table 2 Comparison of Operative Data Between Pleural Effusion and Non-Pleural Effusion Groups

\begin{tabular}{|l|l|l|l|}
\hline & Pleural Effusion (n=64) & Non-Pleural Effusion (n=43) & P-value \\
\hline $\begin{array}{l}\text { Type of donor, n (\%) } \\
\text { - LDLT }\end{array}$ & $63(98.4)$ & $42(97.7)$ & 0.645 \\
Graft-to-weight ratio (\%), median (IQR) & $2.5(1.8,2.8)$ & $2.2(1.8,2.9)$ & 0.789 \\
Operative time (hours), median (IQR) & $11.3(10.3,12.5)$ & $11.5(10.5,13.0)$ & 0.950 \\
\hline
\end{tabular}

Abbreviation: LDLT, living donor liver transplantation. 
Table 3 Comparison of Intraoperative and Postoperative Fluids Between Pleural Effusion and Non-Pleural Effusion Groups

\begin{tabular}{|c|c|c|c|}
\hline & Pleural Effusion $(n=64)$ & Non-Pleural Effusion $(n=43)$ & $P$-value \\
\hline Intraoperative blood loss (mL), median (IQR) & $2250(1100,3750)$ & $1500(700,2700)$ & 0.106 \\
\hline Intraoperative blood loss (mL/kg), median (IQR) & $232.5(100.8,427.5)$ & $129.3(71.4,320.5)$ & 0.877 \\
\hline Intraoperative PRC transfusion $(\mathrm{mL})$, median (IQR) & $880(525,1873)$ & $570(430,1170)$ & 0.120 \\
\hline Intraoperative PRC transfusion (mL/kg), median (IQR) & $94.6(60.7,184.6)$ & $73.3(40.8,103.93)$ & 0.077 \\
\hline Intraoperative blood component transfusion $(\mathrm{mL})$, median (IQR) & $3678(2170,5528)$ & $2888(1900,4658)$ & 0.189 \\
\hline Intraoperative blood component transfusion (mL/kg), median (IQR) & $170.3(96.7,294.6)$ & II $4 . \mid(47.6,226.6)$ & 0.054 \\
\hline Intraoperative fluid balance (mL/kg), median (IQR) & $115.9(63.6,115.9)$ & $124.6(82.0,124.6)$ & 0.754 \\
\hline \multicolumn{4}{|l|}{ Postoperative fluid balance $(\mathrm{mL} / \mathrm{kg}$ ), median (IQR) } \\
\hline - Ist day & $53.6(26.3,83.2)$ & $31.1(10.3,63.0)$ & 0.212 \\
\hline - 2nd day & $20.2(-8.3,54.2)$ & $16.6(-7.7,47.6)$ & 0.363 \\
\hline - 3rd day & $4.2(-21.0,28.7)$ & $-2.9(-25.6,22.1)$ & 0.887 \\
\hline
\end{tabular}

Abbreviation: PRC, packed red cells.

Table 4 Comparison of Postoperative Complications Between Pleural Effusion and Non-Pleural Effusion Groups

\begin{tabular}{|l|l|l|l|}
\hline Complication & Pleural Effusion (n=64) & Non-Pleural Effusion (n=43) & P-value \\
\hline Postoperative complication, n (\%) & $60(93.7)$ & $36(83.7)$ & 0.094 \\
Acute rejection, n (\%) & II (17.2) & $6(13.9)$ & 0.654 \\
Vascular complications, n (\%) & $32(50.0)$ & $19(44.2)$ & 0.555 \\
Bile duct complication, n (\%) & $17(26.6)$ & $4(9.3)$ & $0.028^{*}$ \\
Intra-abdominal bleeding, n (\%) & $8(12.5)$ & $3(7.0)$ & 0.520 \\
Bowel perforation, n (\%) & $6(9.4)$ & 0 & 0.079 \\
Infection, n (\%) & $28(43.7)$ & $13(30.2)$ & 0.159 \\
Abdominal collection, n (\%) & $8(12.5)$ & $6(13.9)$ & 0.827 \\
Reoperation, n (\%) & $35(55.6)$ & $13(30.9)$ & $0.013^{*}$ \\
\hline
\end{tabular}

Note: $* P<0.05$ was statistically significant.

Table 5 Comparison of Postoperative results Between Pleural Effusion and Non-Pleural Effusion Groups

\begin{tabular}{|l|l|l|l|}
\hline Results & Pleural Effusion (n=64) & Non-Pleural Effusion (n=43) & $P$-value \\
\hline Length of ICU stay (days), median (IQR) & $10.0(7.0,20.0)$ & $7.0(6.0,8.0)$ & $0.013^{*}$ \\
Length of hospital stay (days), median (IQR) & $48.0(36.0,63.0)$ & $34.0(27.0,43.0)$ & $0.018^{*}$ \\
Time to extubation (days), median (IQR) & $4.0(3.0,14.0)$ & $3.0(2.0,5.0)$ & 0.059 \\
Oxygen dependence time (days), median (IQR) & $18.5(7.5,33.5)$ & $7.0(5.0,12.0)$ & $0.00 I^{*}$ \\
\hline
\end{tabular}

Note: $* P<0.05$ was statistically significant.

increased risk for post-LT pleural effusion in patients with high PELD score. Moreover, those with severely restrictive pulmonary patterns and poor nutritional status are at risk of operative and postoperative complications. ${ }^{2,12}$

Previous studies demonstrated the intraoperative transfusion $\leq 100 \mathrm{~mL} / \mathrm{kg} /$ day and fluid balance $\leq 14 \mathrm{~mL} / \mathrm{kg} /$ day on the first three days significantly decreased the incidence of pulmonary complications after LT. ${ }^{6,15}$ However, we did not identify a significant difference of fluid balance between the two groups. Moreover, during the first few days after LT, water-sodium retention is aggravated by using high-dose methylprednisolone for the prevention of acute cellular rejection, resulting in positive fluid balance. $^{15}$ Practically, it is difficult to reach a negative fluid balance during the first few days postoperation.

Post-LT pleural effusion is thought to be caused by disruption of diaphragmatic lymphatics during hepatectomy and diaphragmatic injury from prolonged retraction of right hemidiaphragm during operation resulting in fluid shift from abdominal to pleural cavity. ${ }^{2,7}$ Moreover, it can be caused by subdiaphragmatic pathology including infection, hematoma, and biloma. ${ }^{16}$ In our study, majority of available fluid profiles were exudative which was different from previous reports which showed predominant 
Table 6 Comparison of Postoperative Results Between Pleural Effusion Who Required Interventions and Those Who Required No Interventions

\begin{tabular}{|c|c|c|c|}
\hline Results & $\begin{array}{l}\text { Pleural Effusion Without } \\
\text { Interventions }(n=40)\end{array}$ & $\begin{array}{l}\text { Pleural Effusion With } \\
\text { Interventions }(n=24)\end{array}$ & $P$-value \\
\hline Length of ICU stay (days), median (IQR) & $8(6,18)$ & $17(9,26)$ & $0.014^{*}$ \\
\hline Length of hospital stay (days), median (IQR) & $43(33,57)$ & $60(40,73)$ & $0.015^{*}$ \\
\hline Time to extubation (days), median (IQR) & $3(2,10)$ & $12(4,19)$ & $0.004^{*}$ \\
\hline Oxygen dependence time (days), median (IQR) & $10(7,24)$ & $31(25,40)$ & $0.000 *$ \\
\hline
\end{tabular}

Note: $* P<0.05$ was statistically significant.

transudative pleural effusion. ${ }^{7,17}$ Previous studies showed that post-LT pleural effusion requiring intervention was more common with recipients who received split liver donor grafts, ${ }^{11}$ probably due to leakage of blood, bile and lymph from the cut surface of liver graft. ${ }^{11,18}$

Approximately one-third of our patients required therapeutic interventions for pleural effusion. Indications for intervention at our institution included symptoms or signs of compromised respiratory status; and when infectious effusion could not be excluded. Although pleural effusion eventually resolved either with or without therapeutic interventions, it was associated with prolonged oxygen dependence time, length of ICU stay and hospital stay. Most patients who required LT in this study were young children below 3 years of age and suffered from significant malnutrition. Young children were at risk of compromised respiratory status after major abdominal operations particularly when complicated with pleural effusion. These factors could have contributed to prolonged extubation in both groups, particularly in those who required interventions in the pleural effusion group. Therefore, early referral of children with cirrhosis for LT is crucial in order to reduce post-LT morbidity including post-LT pleural effusion.

In our report, six patients unfortunately died but the cause of death was not related to pleural effusion. Other postoperative factors such as graft non-function and infections contributed to patients' morbidity and mortality.

This study had the strength of single institution data which warranted a standardized protocol of patient care. We acknowledge some limitations of the study besides missing data. Many patients were very sick at the time of LT which could have affected the intra- and postoperative outcomes. Therefore, further prospective studies with large sample size would be crucial to confirm our findings.

In conclusion, high PELD score at LT $\geq 18$ is a potential risk factor of pleural effusion following LT.
Post-LT pleural effusion is associated with prolonged oxygen dependence, ICU stay and hospital stay, particularly in those who required interventions. Therefore, early referral of children with cirrhosis for LT is crucial in order to alleviate post-LT pleural effusion resulting in shorter hospital stay.

\section{Acknowledgments}

The authors acknowledge the Ramathibodi Liver Transplantation Team for their great contributions to patient care, Sani Molagool, MD and Assist. Prof. Pornsri Thanachatchairattana, MD, Department of Surgery, Faculty of Medicine Ramathibodi Hospital for their support of the study, and Miss Kornkanok Somboonpun, Department of Epidemiology and Biostatistics, Faculty of Medicine Ramathibodi Hospital, for her assistance with statistical analysis.

\section{Disclosure}

The authors report no conflicts of interest in this work.

\section{References}

1. Glanemann M, Kaisers U, Langrehr JM, et al. Incidence and indications for re-intubation during post-operative care following orthotopic liver transplantation. J Clin Anesth. 2001;13:377-382. doi:10.1016/ S0952-8180(01)00290-2

2. Feltracco P, Carollo C, Barbieri S, Pettenuzzo T, Ori C. Early respiratory complications after liver transplantation. World $J$ Gastroenterol. 2013;19:9271-9281. doi:10.3748/wjg.v19.i48.9271

3. Pirat A, Ozgur S, Torgay A, Candan S, Zeyneloglu P, Arslan G. Risk factors for postoperative respiratory complications in adult liver transplant recipients. Transplant Proc. 2004;36:218-220. doi:10.1016/j. transproceed.2003.11.026

4. Hong SK, Hwang S, Lee SG, et al. Pulmonary complications following adult liver transplantation. Transplant Proc. 2006;38:2979-2981. doi:10.1016/j.transproceed.2006.08.090

5. Ruchonnet-Metrailler I, Blanchon S, Luthold S, et al. Pulmonary complications after liver transplantation in children: risk factors and impact on early post-operative morbidity. Pediatr Transplant. 2018;22 (6):e13243. doi:10.1111/petr.13243

6. Alam S, Sapare A, Rao S, Aggarwal R, D’Cruz AL. Respiratory morbidity following pediatric orthotopic liver transplantation. Indian Pediatr. 2017;54:244-246. 
7. Mack CL, Millis JM, Whitington PF, Alonso EM. Pulmonary complications following liver transplantation. Pediatr Transplant. 2000;4:39-44. doi:10.1034/j.1399-3046.2000.00080.x

8. Sato T, Okamoto K, Sadanaka M, Board J, Mceniery J. High incidence of postoperative pulmonary complications after orthotopic liver transplantation in children. $J$ Anesth. 1994;8:274-276. doi:10.1007/BF02514649

9. Lui JK, Spaho L, Holzwanger E, et al. Intensive care of pulmonary complications following liver transplantation. J Intensive Care Med. 2018;33:595-608. doi:10.1177/0885066618757410

10. Bozbas SS, Eyuboglu FO, Ozturk Ergur F, et al. Pulmonary complications and mortality after liver transplant. Exp Clin Transplant. 2008;6:264-270.

11. Adetiloye VA, John PR. Intervention for pleural effusions and ascites following liver transplantation. Pediatr Radiol. 1998;28:539-543. doi:10.1007/s002470050406

12. Wiering L, Sponholz F, Brandl A, et al. Perioperative pleural drainage in liver transplantation: a retrospective analysis from a high-volume liver transplant center. Ann Transplant. 2020;25: e918456. doi:10.12659/AOT.918456

13. Bourdeaux C, Tri TT, Gras J, et al. PELD score and posttransplant outcome in pediatric liver transplantation: a retrospective study of 100 recipients. Transplantation. 2005;79:1273-1276. doi:10.1097/ 00007890-200505150-00060
14. Barshes NR, Lee TC, Udell IW, et al. The pediatric end- stage liver disease (PELD) model as a predictor of survival benefit and posttransplant survival in pediatric liver transplant recipients. Liver Transplant. 2006;12:475-480. doi:10.1002/1t.20703

15. Jiang GQ, Chen P, Bai DS, Tan JW, Su H, Peng MH. Individualized peri-operative fluid therapy facilitating early-phase recovery after liver transplantation. World J Gastroenterol. 2012;18:1981-1986. doi:10.3748/wjg.v18.i16.1981

16. Spizarny DL, Gross BH, McLoud T. Enlarging pleural effusion after liver transplantation. J Thorac Imaging. 1993;8:85-87. doi:10.1097/ 00005382-199324000-00012

17. Olutola PS, Hutton L, Wall WJ. Pleural effusion following liver transplantation. Radiology. 1985;157(3):594. doi:10.1148/ radiology.157.3.3903852

18. Miraglia R, Maruzzelli L, Piazza M, et al. Real-time ultrasound-guided placement of a pigtail catheter in supine position for draining pleural effusion in pediatric patients who have undergone liver transplantation. J Clin Ultrasound. 2016;44:284-289. doi:10.1002/jcu.22294

\section{Publish your work in this journal}

Transplant Research and Risk Management is an international, peer-reviewed open access journal focusing on all aspects of transplantation and risk management to achieve optimal outcomes in the recipient improving survival and quality of life. The manuscript management system is completely online and includes a very quick and fair peer-review system, which is all easy to use. Visit http:// www.dovepress.com/testimonials.php to read real quotes from published authors. 